\title{
Desmoplastic fibroma of the mandible
}

\begin{abstract}
Introduction: Desmoplastic fibroma (DF) is a rare fibroblastic lesion of bone that histologically resembles the desmoid tumor of soft tissue. It's a nonmetastasizing but locally aggressive tumour.

Clinical case: We report a case of DF of the right mandibular in a 52-year-old male treated with a wide surgical excision.

Discussion: With less than 200 cases reported in literature, DF is a very rare benign bone tumour. In the head and neck region, they are most commonly seen in the mandible and have been treated in various ways. Clinically, DF has a variable symptomatology. Radiographically, it appears as a multilocular or occasionally unilocular radiolucency with well-defined or ill-defined margins. Positive diagnosis is based on surgical pathology. The treatment consists of wide surgical excision. Radiation therapy sometimes has been used. Recurrences will depend on the quality of the treatment.
\end{abstract}

Conclusion: Remaining histology the gold standard for the DF diagnosis.

Keywords: desmoplastic fibroma, benign tumor, mandible, surgical pathology
Volume I Issue I - 2017

\author{
Sinaa Mohamed,' Choumi Faical, ${ }^{2}$ Moumin \\ Mohammed $^{2}$ \\ 'Department of Pathology, Military Hospital Moulay Ismail, \\ Morocco \\ ${ }^{2}$ Department of Oral and Maxillofacial Surgery, Military Hospital \\ Moulay Ismail, Morocco
}

\begin{abstract}
Correspondence: Sinaa Mohamed, Department of Pathology, Military Hospital Moulay Ismail, Meknes, Morocco, Tel
\end{abstract} 00212661185157, Email sinaamohamed57@gmail.com

Received: August 23, 2017| Published: September 06, 2017

\section{Introduction}

Desmoplastic fibroma of bone is a rare myofibroblastic tumor, locally aggressive, benign lesion that histologically resembles a desmoid tumor of the soft-tissue. It was initially described in 1958 by Jaffe, who separated it as a distinct entity from other intraosseous fibrous tumors. This tumor constitutes $<1 \%$ of bone tumors and about $0.3 \%$ of all benign osseous tumors and it usually involves the tibia, scapula and femur. ${ }^{1}$ The mandible represents the fourth most commonly affected site. It has a predilection to affect the posterior part of the mandible, most often in the ramus-angle region. Clinical symptomatology is not specific. The imagery makes it possible to appreciate the extensive potential of the tumor. The histological appearance of the desmoplastic fibroma is identical to that of the extra-osseous desmoid, although the fibroma is infiltrative; there are no mitoses or nuclear atypia. The treatment is surgical and the quality of this treatment condition the occurrence of recurrences. ${ }^{1,2}$

\section{Case report}

A 52 year-old male consulted for a right mandibular tumor which has been progressively of 14 month duration. The clinical examination revealed an enormous right mandibular tumor, deforming the face (Figure 1). The endobuccal examination objectivated a bud mucosal tumor in the right molar region. Computed tomography (CT) showed a unilocular and hypodense lesion of the right hemimandibule, lysing the cortical, without reaction periosteal (Figure 2). A biopsy has been performed, it wasn't conculant. The patient has benefited of right hemimandibulectomy. The macroscopic examination showed a mandiblar mass measuring $13 \times 7 \times 5 \mathrm{~cm}$ with a firm consistance (Figure 3 ). The histological study showed a benign proliferation formed by interlaced bundles and whorled aggregates of densely fibroblastic collagenous matrix, containing uniform spindle and elongated fibroblasts. The cellularity is low, with some foci of hypercellularity. No cytologic atypia or mitotic figures neither osseous matrix is observable (Figure 4). The diagnosis of a desmoplastic fibroma was retained. The controle for 2 years didn't show any signs of recurrence.

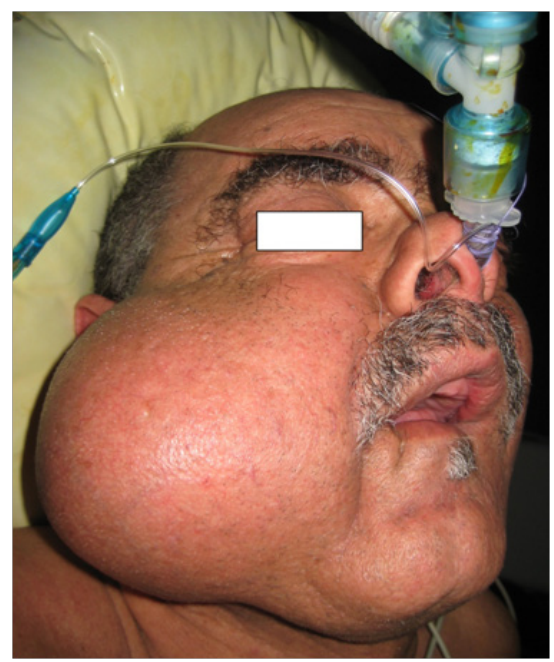

Figure I Photograph showing an enormous right mandibular tumor.

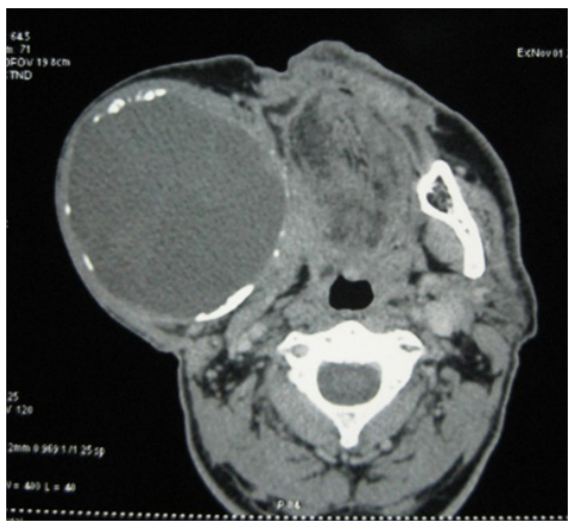

Figure 2 CT scans in axial view. A uniloculated and hypodense mass subverts the structure of the right mandible, lysing the cortical, without reaction periostal (arrowheads). 


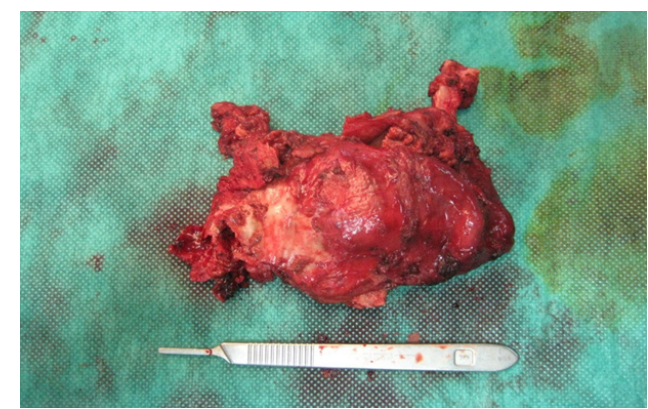

Figure 3 Photograph showing the surface of the surgical specimen.

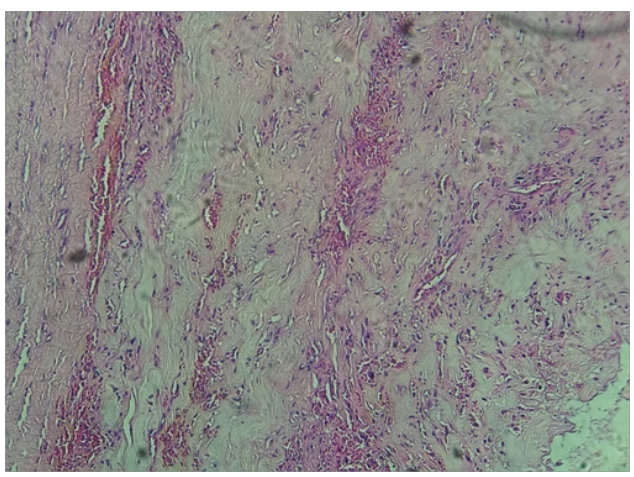

Figure 4 Photomicrograph showing monomorphic, wavy, spindled cells with moderate cellularity and elongated nuclei, without cellular pleomorphism or mitoses, in abundant dense matrix of collagen ( $\mathrm{H} \& \mathrm{E} \times 200$ magnification).

\section{Discussion}

Griffith and Irby reported in 1965 the first involvement of a DF involving the mandible. Up to the present, about 180 of DF of mandible cases have been reported in literature. The WHO defines a desmoplastic fibroma as a rare, benign bone tumour composed of spindle cells with minimal atypia and abundant collagen production. ${ }^{1}$ They account for approximately $0.1 \%$ of all primary bone tumours in the body. DF can involve any bone but is most often found in the mandible $(22 \%)$, followed by the femur (15\%), pelvic bones $(13 \%)$, radius $(12 \%)$, and tibia $(9 \%) .74 \%$ of the patients were younger than 31 years and only $6 \%$ were older than 50 years. ${ }^{1-3}$

While exact etiologic factors for the DF currently remain unknown, possible association with trauma, endocrine factors, genetic aberrations, or multifactorial etiology have been suggested. ${ }^{3}$

Painless and slow-growing are the most common symptom (72\%), followed by facial asymmetry (20\%), tooth displacement and/ or root divergence $(12 \%)$, history of previous trauma to the affected area $(13 \%)$, limited mandibular opening or trismus (7\%), mimicking odontogenic infection (6\%) and tooth mobility (5\%). However, $6.3 \%$ of patients did present with a rapidly enlarging mass. ${ }^{3,4}$

Radiographically, Computed tomography (CT) or magnetic resonance imaging (MRI) is preferred over routine $\mathrm{X}$-ray imaging. The radiographic features of DF are non specific. A well demarcated lytic lesion is seen. It is usually multilocular and often expands the bone. The radiographic differential diagnosis includes ameloblastoma, odontogenic fibroma, aneurysmal bone cyst and hemangioma. Only rarely will primary malignant lesions such as fibrosarcoma or malignant fibrous histiocytoma be suspected on the basis of radiographic evidence. ${ }^{5}$
A DF often behaves in an aggressive manner and macroscopically has a firm consistency with well-defined advancing surfaces that may extend into surrounding soft tissue. The histological features of DF and the extra-abdominal desmoid tumour are essentially identical. They are characterized by uniform-appearing fibroblastic cells in a stroma containing various amounts of collagen fibers. The morphologic differential diagnosis includes benign and malignant spindle cell tumours of bone. Fibrous dysplasia can stimulate desmoplastic fibroma in areas where fibrous tissue predominates and osteoid production is not apparent. The distinction can be made by recognizing areas of bone formation by additional sampling. Also the nuclei in fibrous dysplasia are shorter and more compact-looking than the elongated, slender nuclei seen in desmoplastic fibroma. Low grade intraosseous osteosarcoma, another tumour that can mimic desmoplastic fibroma, can also be excluded by identification of bone formation.

Nonossifying fibroma and solitary congenital fibromatosis of bone can be confused with desmoplastic fibroma. Low grade fibrosarcoma poses the most difficult problem in the histological differential diagnosis; in fact, the distinction may not always be possible and can only be detected when it recurs and metastasizes. However, fibrosarcoma is more cellular, with a recognizable herringbone pattern and plumper, larger cells than those in desmoplastic fibroma. Cytologically hyperchromasia with anaplasia and mitotic activity quantitatively surpasses the rare mitotic figures occasionally seen in desmoplastic fibroma. ${ }^{6-9}$

Jaffe, in his discussion of the treatment of desmoplastic fibroma of bone, recommended segmental resection as the treatment of choice and noted that if the lesion is curetted and recurs, segmental resection or a more thorough curettage should be performed..$^{10}$ Wide resection or a thorough "marginal" curettage was the preferred method of treatment while local or limited curettage often led to continued growth of the tumour. There are conflicting reports regarding the role of radiotherapy in the management of desmoid tumours.

In 1944, Pack and Ehrlich, stated that radiation therapy could affect regression of desmoid tumours, but this process was slow. ${ }^{11}$ Other authors, even recently, have judged radiation to be of limited value in the curative treatment of patients with desmoid tumours. Radiation therapy is recommended in those situations where widefield resection without significant morbidity is not possible for gross local disease. Role of chemotherapy and hormonal therapy in the management of desmoid tumours is not clear., ${ }^{9,10,12}$

\section{Conclusion}

Desmoplastic fibroma is a rare intraosseous well-differentiated benign fibrous tumour, but locally aggressive. Distinguishing DF from other spindle cells proliferations has important prognostic significance and remains a difficult process for the pathologist. Wide resection or en bloc resection with long-term follow-up continues to be the treatment of choice.

\section{Acknowledgements}

None.

\section{Conflict of interest}

The author declares no conflict of interest. 


\section{References}

1. Averna R, De Filippo M, Ferrari S, et al. Desmoplastic fibroma of the mandible. Acta Biomed. 2011;82(1):69-73.

2. Woods TR, Cohen DM, Islam MN, et al. Desmoplastic Fibroma of the Mandible: a series of three cases and review of literature. Head Neck Pathol. 2015;9(2):196-204.

3. Pınar Sumer, Mahmut Sumer, Armağan Çalışkan, et al. Desmoplastic fibroma of the mandible: a case report. Clinical Dentistry and Research. 2016;40(2):73-78.

4. Schneider M, Zimmermann AC, Depprich RA, et al. Desmoplastic fibroma of the mandible - review of the literature presentation of a rare case. Head Face Med. 2009;5:25.

5. Shi H, Wang P, Wang S, et al. Desmoplastic fibroma of the mandible. Dentomaxillofacial Radiology. 2008;37:408-411.

6. Lt Col VK Shukul, Wg Cdr S Saxena, Dr BG Shankar. Desmoplastic Fibroma: Mandible. MJAFI. 2004;60:307-309.
7. Benazzou S, Boulaadas M, Hajji F, et al. Mandibular desmoplastic fibroma: Knowing when to consider it! Med Buccale Chir Buccale. 2010;21(1):31-35

8. Yadavalli Guruprasad, Dinesh Singh Chauhan. Desmoplastic fibroma of mandible. Medical Journal of Dr. DY Patil University. 2015;8(2):254 257.

9. Reid EN, Lawoyin DO, Suresh L, et al. Desmoplastic fibroma of the anterior mandible. Case report and review of literature. $N$ Y State Dent J. 2009;75(3):32-33.

10. Jaffe HL. Tumors and tumorous conditions of the bones and joints. Philadelphia, Pa. Lea \& Febiger. 1958;12(1):298-303.

11. Kiel KD, Suit HD. Radiation therapy in the treatment of aggressive fibromatosis (Desmoid tumours). Cancer. 1984;54(10):2051-2055.

12. De Bree E, Zoras O, Hunt JL, et al. Desmoid tumors of the head and neck: a therapeutic challenge. Head Neck. 2014;36(10):1517-1526. 